\title{
GMR
}

\section{Developmental methylation pattern regulates porcine GPR120 expression}

\author{
H.M. Wang*, J.D. Ma*, L. Jin, Y.H. Liu, T.D. Che, M.Z. Li and X.W. Li \\ Institute of Animal Genetics and Breeding, \\ College of Animal Science and Technology, \\ Sichuan Agricultural University, Ya'an, Sichuan, China \\ *These authors contributed equally to this study. \\ Corresponding authors: M.Z. Li / X.W. Li \\ E-mail: mingzhou.li@163.com / xuewei.li@sicau.edu.cn \\ Genet. Mol. Res. 15 (1): gmr.15014240 \\ Received September 24, 2015 \\ Accepted November 25, 2015 \\ Published February 5, 2016 \\ DOI http://dx.doi.org/10.4238/gmr.15014240
}

\begin{abstract}
DNA methylation is an important component of the epigenetic machinery and plays a critical role in transcriptional regulation. It mostly occurs in $\mathrm{CpG}$ abundant regions, known as $\mathrm{CpG}$ islands (CGIs). G protein-coupled receptor 120 (GPR120) functions as an omega-3 fatty acid receptor and is involved in multiple-biological processes, including lipogenesis. Herein, we show that GPR120 is highly expressed in porcine mature adipose tissue and is positively associated with adipose tissue development $(r=0.86, P<0.01)$. We also predicted 5 CGls across the GPR120 genomic sequence and investigated their methylation status using the MassArray approach. Our results show that these CGls exhibit significantly different methylation states $\left(P_{C G I}<0.01\right)$, and that the DNA methylation of GPR1205'-untranslated and first exon regions can negatively regulate its expression levels. This study will aid further investigations on the epigenetic mechanism regulating GPR120 expression.
\end{abstract}

Key words: GPR120; Methylation; CpG islands; Adipose tissue; Pig 
H.M. Wang et al.

\section{INTRODUCTION}

Adipose tissues are the principal storage sites for lipids in mammals. Adipocytes constitute the main cellular component of adipose tissue and are the chief storage sites of energy in the form of triglyceride droplets. Small adipocytes avidly absorb free fatty acids in the postprandial period and act as a sink or powerful buffer to free fatty acid levels (Ibrahim, 2010). Free fatty acids provide an important energy source and act as signaling molecules in various cellular processes (Itoh et al., 2003). As free fatty acid receptors, several G-protein-coupled receptors are important in adipose tissue physiology as well as in several adipose tissue-related diseases (Hirasawa et al., 2005; Steneberg et al., 2005; Maslowski et al., 2009).

G protein-coupled receptor 120 (GPR120) functions as an omega-3 fatty acid receptor and plays critical roles in various physiological processes, including the adjustment of adipogenesis and whole-body lipid metabolism, especially fat distribution (Oh et al., 2010; Ichimura et al., 2012). GPR120 is the only highly expressed G protein-coupled receptor in both human and mouse mature adipose tissues, and it promotes adipogenesis by enhancing glucose uptake in adipocytes (Gotoh et al., 2007; Oh et al., 2010). GPR120 is abundantly expressed in the porcine intestine (Colombo et al., 2012), but the role of GPR120 in porcine adipose tissue is poorly understood. It is worth exploring whether GPR120 is a potential candidate gene for the pig fat mass trait.

DNA methylation is a crucial epigenetic modification involved in various biological functions, including adipogenesis, and mostly occurs in CpG abundant regions known as CpG islands (CGIs) (Sakamoto et al., 2008; Zhu et al., 2012). Previous reports indicate that the function of DNA methylation varies depending on the location of methylation within a gene, such as untranslated or coding regions (Jones, 2012). In most cases, 5'-untranslated region (5'-UTR) methylation is negatively correlated with gene transcription (Eckhardt et al., 2006), while intragenic methylation shows a positive correlation with gene transcription (Jjingo et al., 2012; Lorincz et al., 2004). In addition, it has been reported that hypermethylation of the p15 INK4b $3^{\prime}-$ UTR interferes with its transcriptional regulation (Malumbres et al., 1999). Nonetheless, the epigenetic mechanisms that control the expression of GPR120 remain to be determined.

The pig is an ideal biomedical model for adipose tissue development as humans and pigs are similar with regard to whole body size and metabolism (Lunney, 2007; Schook et al., 2005). Herein, we elucidate the role of the porcine GPR120 gene in promoting porcine adipose tissue development. We also determined the methylome landscape in the GPR120 gene. This study adds to our understanding of the role of GPR120 in porcine adipose physiology and will aid in the search for epigenetic biomarkers of GPR120 regulation.

\section{MATERIAL AND METHODS}

\section{Experimental animal and tissue samples}

All Jinhua female pigs were fed a normal diet in accordance with Chinese local pig rearing standards; animals had access to feed and water ad libitum and lived under typical conditions. Subcutaneous adipose tissues (SATs) were obtained from Jinhua female pigs at 3 developmental stages (i.e., birth, 30, and 180 days), and we used 3 pigs as biological repeats at each stage. All samples were stored at $-80^{\circ} \mathrm{C}$ until RNA and DNA extraction. 


\section{Histology of adipocytes}

Measurement of porcine adipocyte volume was performed as previously described (Li et al., 2012). All SATs were fixed in $10 \%$ neutral buffered formalin solution after sacrifice, embedded in paraffin using a TP1020 semi-enclosed tissue processor (Leica), sliced at a thickness of $6 \mu \mathrm{m}$ by using the RM2135 rotary microtome (Leica), and stained with hematoxylin and eosin. One hundred cells were measured for each sample in randomly selected fields using a TE2000 fluorescence microscope (Nikon) and the Image Pro-Plus 7.0 software 27 (Media-Cybernetics). Then, the mean diameter of an adipocyte cell was calculated as the geometric average of the maximum and minimum diameter. The mean adipocyte volume $(\mathrm{V})$ was obtained according to the following formula:

$$
\mathrm{V}=\pi / 6 \mathrm{Sf}_{\mathrm{i}} \mathrm{D}_{\mathrm{i}}^{3} / \mathrm{Sf}_{\mathrm{i}}
$$

where $D_{i}$ is the mean diameter; and $f_{i}$ denotes the number of cells with that mean diameter $D_{i}$.

\section{Quantitative real-time reverse transcription-polymerase chain reaction (qRT-PCR)}

Total RNA was isolated from the adipose tissues using TRIzol reagent (TaKaRa, Dalian, China) according to the manufacturer protocol. Total RNA was reverse transcribed to complementary DNA (cDNA) using the oligo (dT) and random 6-mers primers provided in the PrimeScript RT Master Mix kit (TaKaRa), following the manufacturer recommendation. The qRT-PCR was performed using the SYBR Premix Ex Taq kit (TaKaRa) on a CFX96 RealTime PCR detection system (Bio-Rad, Richmond, CA, USA). The gene-specific primers are shown in Table 1. Porcine $A C T B, T B P$, and $T O P 2 B$ were simultaneously used as endogenous control genes. The relative expression levels of objective mRNAs were calculated using the $\Delta \Delta$ Ct method.

\begin{tabular}{|c|c|c|c|}
\hline Gene symbol & Primer sequence ( $5^{\prime}$ to $\left.3^{\prime}\right)$ & GenBank ID & Amplicon (bp) \\
\hline \multirow{2}{*}{ GPR120 } & F: TCCAGAACTTCAAGCAAAACCT & \multirow{2}{*}{ NM_001204766.1 } & \multirow{2}{*}{253} \\
\hline & R: GTGACAAATAGATGCCGATAGACAA & & \\
\hline \multirow{2}{*}{ ACTB } & F: TCTGGCACCACACCTTCT & \multirow{2}{*}{ DQ178122 } & \multirow{2}{*}{114} \\
\hline & R: TGATCTGGGTCATCTTCTCAC & & \\
\hline \multirow{2}{*}{ TBP } & F: GATGGACGTTCGGTTTAGG & \multirow{2}{*}{ DQ178129 } & \multirow{2}{*}{124} \\
\hline & R: AGCAGCACAGTACGAGCAA & & \\
\hline \multirow{2}{*}{ TOP2B } & F: AACTGGATGATGCTAATGATGCT & \multirow{2}{*}{ AF222921 } & \multirow{2}{*}{137} \\
\hline & R: TGGAAAAACTCCGTATCTGTCTC & & \\
\hline
\end{tabular}

ACTB ( $\beta$ actin), TBP (TATA box binding protein) and TOP2B (topoisomerase II $\beta$ ) are the endogenous control genes. $\mathrm{F}$ indicates forward primers, and $\mathrm{R}$ indicates reverse primers.

\section{CpG island prediction}

The GPR120 gene sequence (2000 bp upstream of the first exon to 2000 bp downstream 
of the last exon) was download from Ensembl Genome Browser (Sscrofa 10.2) and scanned for the distribution of $\mathrm{CpG}$ islands using the $\mathrm{CpG}$ island searcher online software (http://cpgislands. usc.edu/) with the parameters set as lower limits: $\% \mathrm{GC}=55, \mathrm{ObsCpG} / \mathrm{ExpCpG}=0.65$, length $=$ $200 \mathrm{bp}$, distance $=100 \mathrm{bp})$.

\section{DNA methylation sequencing by MassArray}

Four pairs of primers spanning predicted CpG islands (CGI1, CGI2, CGI3, and CGI4) of porcine GPR120 were designed using the EpiDesigner software (http://www.epidesigner.com); 2 successful primer pairs were designed for the 5'-UTR and whole exon 1 regions of CGI3. Primer sequences are listed in Table 2. The genomic DNA was isolated using the DNeasy Blood \& Tissue Kit (Qiagen, Hilden, Germany). Subsequent bisulfite treatment of genomic DNA was performed using the EZ DNA methylation-Gold Kit (Zymo Research, Irvine, CA, USA) following the manufacturer protocol. Quantitative methylation analyses of the CpG sites were performed using the Sequenom MassArray platform (CapitalBio, Beijing, China) as previously described ( $\mathrm{Li}$ et al., 2012). The resultant methylation calls were analyzed with the EpiTyper software v1.0 (Sequenom) to generate quantitative results for each CpG or an aggregate of multiple CpGs.

\begin{tabular}{|c|c|c|c|c|}
\hline \multirow[b]{2}{*}{ Name } & \multirow[b]{2}{*}{ Primer sequence ( $5^{\prime}$ to $\left.3^{\prime}\right)$} & \multicolumn{3}{|c|}{ Amplicon } \\
\hline & & $\begin{array}{l}\text { CpG } \\
\text { island } \\
\text { ordinal }\end{array}$ & $\begin{array}{l}\text { Size } \\
\text { (bp) }\end{array}$ & $\begin{array}{l}\text { No. of } \\
\text { CpGs } \\
\text { covered }\end{array}$ \\
\hline \multirow{2}{*}{ GPR120-1 } & F: aggaagagagATGATTTGTGTGTGTATTTGGAGTG & \multirow{2}{*}{ CGI1 } & \multirow{2}{*}{487} & \multirow{2}{*}{15} \\
\hline & R: cagtaatacgactcactatagggagaaggctAACACACAATAATTTTCCTAAAACCTC & & & \\
\hline \multirow{2}{*}{ GPR120-2 } & F: aggaagagagTTAGATTTAGGGGGTTTTTTTTGAT & \multirow{2}{*}{ CGI2 } & \multirow{2}{*}{413} & \multirow{2}{*}{13} \\
\hline & R: cagtaatacgactcactatagggagaaggctAAACACCCAAAAATACAAAATCCTT & & & \\
\hline \multirow{2}{*}{ GPR120-3 } & F: aggaagagagTTGGGGGTTTATTAGATAAGGTTTT & \multirow{2}{*}{ CGI3 } & \multirow{2}{*}{454} & \multirow{2}{*}{36} \\
\hline & R: cagtaatacgactcactatagggagaaggctCCACAAAAATAAATACCAACACCAC & & & \\
\hline \multirow{2}{*}{ GPR120-4 } & F: aggaagagagTGGTGTTGGTATTTATTTTTGTGGT & \multirow{2}{*}{ CGI3 } & \multirow{2}{*}{477} & \multirow{2}{*}{46} \\
\hline & R: cagtaatacgactcactatagggagaaggctACCCTTTCCAAACACCTACCTAAC & & & \\
\hline \multirow{2}{*}{ GPR120-5 } & F: aggaagagagGGGATTGTTGGGTAATTTTTTTAAT & \multirow{2}{*}{ CGI4 } & \multirow{2}{*}{411} & \multirow{2}{*}{17} \\
\hline & R: cagtaatacgactcactatagggagaaggctAACCAAACCCAAAATACATATCACT & & & \\
\hline \multirow{2}{*}{ GPR120-6 } & F: aggaagagagTGGTATGTGGAGGTTTTTAGGTTAG & \multirow{2}{*}{ CGI5 } & \multirow{2}{*}{376} & \multirow{2}{*}{10} \\
\hline & R: cagtaatacgactcactatagggagaaggctATCCСАCAAATAAACAAACСТСТTT & & & \\
\hline
\end{tabular}

\section{Statistical analysis}

The data were subjected to ANOVA. Pearson's correlation was used to determine the correlation of the GPR120 transcription levels with the adipocyte volumes or methylation status. The results are reported as means $\pm S D(N=3)$, and $P$ values $<0.05$ were considered statistically significant. All statistical analyses were performed in IBM SPSS Statistics 19 (SPSS 19.0; IBM Corporation, 2010, Armonk, NY, USA). 


\section{RESULTS}

\section{GPR120 promotes porcine adipose tissue development}

The expression of GPR120 in SATs and the adipocyte volume in porcine SATs gradually increased during porcine postnatal development (Figure $1 \mathrm{~A}$ and $\mathrm{B}$, respectively). The expression of GPR120 mRNA was higher in the SATs at 180 days when compared to those at 30 days and birth. Pearson's correlation analysis showed that the mRNA abundance of GPR120 had a clear positive correlation $(r=0.86, P<0.01)$ with adipocyte volume (Figure $1 C)$, which is consistent with known GPR120 biological function that promotes lipogenesis (Gotoh et al., 2007). These results highlight the critical role of GPR120 in the development of porcine adipose tissues.

A

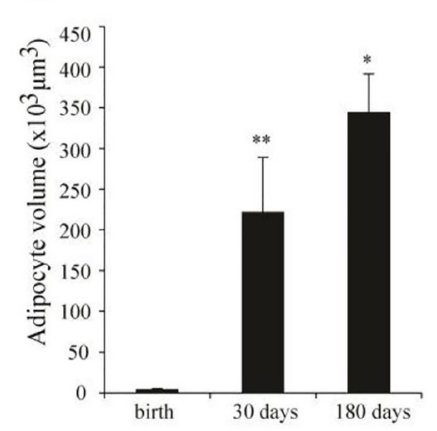

B

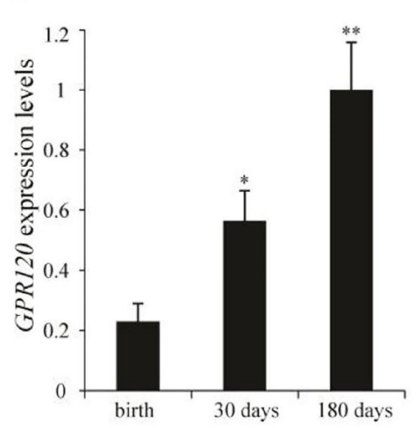

$\mathrm{C}$



Figure 1. Adipocyte volumes and expression levels of GPR120 in subcutaneous adipose tissues (SATs) during porcine postnatal development. A. Mean volumes of SAT at different time points $(\mathrm{N}=3)$. B. Quantification of GPR120 expression in SATs at different development stages $(\mathrm{N}=3)$ by quantitative real-time reverse transcription polymerase chain reaction. C. Relationship between adipocyte volumes and GPR120 expression levels. All results were analyzed using ANOVA. All data are reported as means \pm standard deviation (SD); ${ }^{*} P<0.05,{ }^{* *} \mathrm{P}<0.01$.

\section{Methylation of location-specific CpG islands}

DNA methylation, which mostly occurs in CGIs, is an essential mechanism for the control of gene expression. There is no available annotation for the transcription of the start and end sites of porcine GPR120; therefore, the 5'-UTR was arbitrarily defined as the region within 2000 bp upstream of the first exon, and the 3'-UTR was arbitrarily defined as the region within $2000 \mathrm{bp}$ downstream of the last exon. We analyzed the CGIs across the whole GPR120 sequence (i.e., 2000 bp upstream of the first exon to 2000 bp downstream of the last exon) using the CpG Island Searcher (http://cpgislands.usc.edu/; see Methods). As shown in Figure 2A, 5 predicted CGIs were classified into 4 classes (i.e., 5'-UTR, 5'-UTR-exon 1-intron 1, intron 2, and 3'-UTR) according to their genomic locations. This result suggests that the methylation of GPR120 gene may play important roles in gene regulation.

To investigate the role of methylation in the 5 predicted CGIs, we detected their methylation status in SATs during different developmental stages using the MassArray 
approach (Ehrich et al., 2005). Of the 137 CpG sites identified, 96 were considered informative and suitable for analysis. As shown in Figure 2B, our results revealed significant differences among the 5 CGIs (two-way ANOVA, $P_{C G I}<0.01$ ). Of all the CGIs, low levels of DNA methylation were detected in CGI3 (0.16, 5'-UTR-exon 1), while CGI2 (0.61, the 5'-UTR and close to the first exon), CGI4 (0.78, intron 2), and CGI5 (0.75, 3'-UTR) displayed relatively elevated levels of DNA methylation when compared to that of CGI3. In addition, hypermethylation was present in CGI1 (88\%, 5'-UTR and distal from the first exon). Our findings demonstrate that the location-specific CGIs of GPR120 exhibit significantly different methylation patterns.

\section{A}



$\mathrm{B}$



$\mathrm{C}$

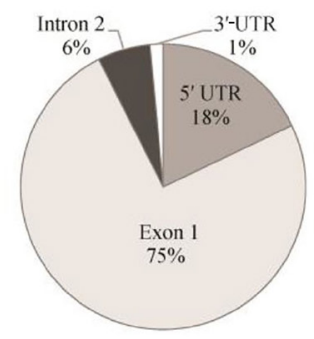

Figure 2. DNA methylation analysis of the porcine GPR120 gene. A. Schematic diagram of CpG islands in the complete genomic region of the porcine GPR120 gene. The black boxes indicate the exons of porcine GPR120, and the red lines represent the predicted $\mathrm{CpG}$ islands. B. Methylation status of $5 \mathrm{CpG}$ islands $(\mathrm{N}=3)$. C. Distribution of differentially methylated sites. All results were analyzed using two-way analysis of variance (ANOVA). All data are reported as means \pm standard deviation (SD).

\section{Effects of differential methylation of CpG sites (DMSs) on GPR120 expression}

We identified $71 \%$ of DMSs ( $<<0.05$; Table S1), and these DMSs were primarily located in the exon 1 and the 5 '-UTR regions (Figure $2 \mathrm{C}$ ). Regression analysis demonstrated that $35 \%$ of DMSs were significantly correlated $(P<0.05)$ with the transcription levels of GPR120 during porcine adipose tissue development (Table S1). Strikingly, the DMSs within the first exon and 5'-UTR were significantly and negatively correlated with GPR120 transcription (Table 3). 
Table 3. Differentially methylated sites that exhibited significant correlations with GPR120 expression levels.

\begin{tabular}{|c|c|c|c|c|c|c|}
\hline \multirow{2}{*}{ Gene elements } & \multirow{2}{*}{ Location } & \multirow{2}{*}{ CpG sites } & \multicolumn{3}{|c|}{ Mean methylation } & \multirow{2}{*}{$r$ value } \\
\hline & & & 180 days & 30 days & Birth & \\
\hline \multirow{7}{*}{ 5'-UTR } & \multirow{2}{*}{ CGI1 } & GPR120-1_CpG_11 & 0.68 & 0.76 & 0.85 & $-0.92^{* *}$ \\
\hline & & GPR120-1 CpG 12 & 0.77 & 0.84 & 0.93 & $-0.90^{* *}$ \\
\hline & CGI2 & GPR120-2_CpG_13 & 0.50 & 0.57 & 0.77 & $-0.90^{* *}$ \\
\hline & \multirow{13}{*}{ CGI3 } & GPR120-3_CpG_12 & 0.00 & 0.02 & 0.11 & $-0.87^{* *}$ \\
\hline & & GPR120-3_CpG_13 & 0.23 & 0.27 & 0.30 & $-0.94^{\star *}$ \\
\hline & & GPR120-3_CpG_14.15 & 0.09 & 0.08 & 0.21 & $-0.75^{* *}$ \\
\hline & & GPR120-3 CpG 16 & 0.00 & 0.02 & 0.11 & $-0.87^{* *}$ \\
\hline \multirow{9}{*}{ Exon 1} & & GPR120-3_CpG_30.31.32 & 0.00 & 0.21 & 0.22 & $-0.88^{* *}$ \\
\hline & & GPR120-3_CpG_34 & 0.04 & 0.02 & 0.09 & $-0.61^{*}$ \\
\hline & & GPR120-3 CpG 36 & 0.04 & 0.02 & 0.09 & $-0.61^{*}$ \\
\hline & & GPR120-3_CpG_4 & 0.00 & 0.03 & 0.06 & $-0.95^{\star *}$ \\
\hline & & GPR120-4 CpG 10.11 & 0.41 & 0.43 & 0.78 & $-0.82^{* \star}$ \\
\hline & & GPR120-4_CpG 12 & 0.68 & 0.65 & 0.84 & $-0.70^{*}$ \\
\hline & & GPR120-4_CpG_21 & 0.68 & 0.65 & 0.84 & $-0.70^{*}$ \\
\hline & & GPR120-4 CpG 35.36 .37 & 0.18 & 0.19 & 0.58 & $-0.80^{* *}$ \\
\hline & & GPR120-4 CpG 38.39 & 0.12 & 0.13 & 0.02 & $0.72^{*}$ \\
\hline
\end{tabular}

${ }^{*} P<0.05 ;{ }^{* *} P<0.01$

\section{DISCUSSION}

In this study, we revealed that the GPR120 gene is incresingly expressed in porcine adipose tissues along with adipose tissue development. We also showed that GPR120 expression is significantly and positively correlated with adipocyte volume. Taken together, these results indicate the important role of GPR120 in porcine adipogenesis, consistent with pravious investigations (Miyauchi et al., 2009). Methylation analysis of CGls across the whole GPR120 sequence showed that methylation is widely but differentially appeared in distinct location of the GPR120 gene, including 5'-UTR, 5'-UTR-exon 1-intron 1, intron 2, and 3'-UTR. Interestingly, CGI3, which covers the entire first exon, may have functional motifs that confer hypomethylation (Brenet et al., 2011; Li et al., 2012). It is well established that DNA methylation of the first exon is tightly linked to transcriptional silencing, and a gene with methylation change in the 5'-UTR, generally, exhibited an inverse correlation between the level of DNA methylation and gene expression (Eckhardt et al., 2006; Brenet et al., 2011; de la Rica et al., 2013). Regression analysis in this study also demonstrated that some of DMSs were significantly correlated $(P<0.05)$ with the transcription levels of GPR120 during porcine adipose tissue development, particular the DMSs within the first exon and 5'-UTR.

\section{CONCLUSIONS}

In the present study, we demonstrated that GPR120 is highly expressed in porcine mature adipose tissue and is involved in porcine adipose tissue development. Therefore, GPR120 may be a candidate gene for the pig fat mass trait. Moreover, to understand the epigenetic mechanism regulating GPR120 expression, we examined the methylation status of CpG islands within the whole GPR120 genomic sequence in SATs across 3 developmental stages. Our results indicate that methylation of the GPR120 5'-UTR and first exon regions were significantly and negatively correlated with gene transcription. Collectively, ours study provides a solid foundation for exploring the epigenetic mechanisms regulating GPR120. 


\section{ACKNOWLEDGMENTS}

Research supported by grants from the National High-Technology Research and Development Program of China ("863" Program; \#2013AA102502), the National Special Foundation for Transgenic Species of China (\#2014ZX0800950B and \#2011ZX08006-003), the Specialized Research Fund of Ministry of Agriculture of China (\#NYCYTX-009), and the Project of Provincial Twelfth Five Years' Animal Breeding of Sichuan Province (\#2011YZGG15).

\section{REFERENCES}

Brenet F, Moh M, Funk P, Feierstein E, et al. (2011). DNA methylation of the first exon is tightly linked to transcriptional silencing. PLoS One 6: e14524. http://dx.doi.org/10.1371/journal.pone.0014524

Colombo M, Trevisi P, Gandolfi G and Bosi P (2012). Assessment of the presence of chemosensing receptors based on bitter and fat taste in the gastrointestinal tract of young pig. J. Anim. Sci. 90: 128-130. http://dx.doi.org/10.2527/jas.53793

de la Rica L, Urquiza JM, Gómez-Cabrero D, Islam AB, et al. (2013). Identification of novel markers in rheumatoid arthritis through integrated analysis of DNA methylation and microRNA expression. J. Autoimmun. 41: 6-16. http://dx.doi.org/10.1016/j.jaut.2012.12.005

Eckhardt F, Lewin J, Cortese R, Rakyan VK, et al. (2006). DNA methylation profiling of human chromosomes 6, 20 and 22. Nat. Genet. 38: 1378-1385. http://dx.doi.org/10.1038/ng1909

Ehrich M, Nelson MR, Stanssens P, Zabeau M, et al. (2005). Quantitative high-throughput analysis of DNA methylation patterns by base-specific cleavage and mass spectrometry. Proc. Natl. Acad. Sci. U. S. A. 102: 15785-15790. http://dx.doi.org/10.1073/pnas.0507816102

Gotoh C, Hong YH, Iga T, Hishikawa D, et al. (2007). The regulation of adipogenesis through GPR120. Biochem. Biophys. Res. Commun. 354: 591-597. http://dx.doi.org/10.1016/j.bbrc.2007.01.028

Hirasawa A, Tsumaya K, Awaji T, Katsuma S, et al. (2005). Free fatty acids regulate gut incretin glucagon-like peptide-1 secretion through GPR120. Nat. Med. 11: 90-94. http://dx.doi.org/10.1038/nm1168

IBM Corporation Released (2010). IBM SPSS Statistics for Windows. Version 19.0. IBM Corp., Armonk.

Ibrahim MM (2010). Subcutaneous and visceral adipose tissue: structural and functional differences. Obes. Rev. 11: 11-18. http://dx.doi.org/10.1111/j.1467-789X.2009.00623.x

Ichimura A, Hirasawa A, Poulain-Godefroy O, Bonnefond A, et al. (2012). Dysfunction of lipid sensor GPR120 leads to obesity in both mouse and human. Nature 483: 350-354. http://dx.doi.org/10.1038/nature10798

Itoh $\mathrm{Y}$, Kawamata $\mathrm{Y}$, Harada M, Kobayashi M, et al. (2003). Free fatty acids regulate insulin secretion from pancreatic $b$ cells through GPR40. Nature 422: 173-176. http://dx.doi.org/10.1038/nature01478

Jjingo D, Conley AB, Yi SV, Lunyak VV, et al. (2012). On the presence and role of human gene-body DNA methylation. Oncotarget 3: 462-474. http://dx.doi.org/10.18632/oncotarget.497

Jones PA (2012). Functions of DNA methylation: islands, start sites, gene bodies and beyond. Nat. Rev. Genet. 13: 484-492. http://dx.doi.org/10.1038/nrg3230

Li M, Wu HL, Luo ZG, Xia YD, et al. (2012). An atlas of DNA methylomes in porcine adipose and muscle tissues. Nat. Commun. 3: 850 . http://dx.doi.org/10.1038/ncomms 1854

Lorincz MC, Dickerson DR, Schmitt M and Groudine M (2004). Intragenic DNA methylation alters chromatin structure and elongation efficiency in mammalian cells. Nat. Struct. Mol. Biol. 11: 1068-1075. http://dx.doi.org/10.1038/nsmb840

Lunney JK (2007). Advances in swine biomedical model genomics. Int. J. Biol. Sci. 3: 179-184. http://dx.doi.org/10.7150/ijbs.3.179

Malumbres M, Perez de Castro I, Santos J, Fernandez Piqueras J, et al. (1999). Hypermethylation of the cell cycle inhibitor p15INK4b 3'-untranslated region interferes with its transcriptional regulation in primary lymphomas. Oncogene 18: 385396. http://dx.doi.org/10.1038/sj.onc.1202299

Maslowski KM, Vieira AT, $\mathrm{Ng} \mathrm{A}$, Kranich J, et al. (2009). Regulation of inflammatory responses by gut microbiota and chemoattractant receptor GPR43. Nature 461: 1282-1286. http://dx.doi.org/10.1038/nature08530

Miyauchi S, Hirasawa A, Iga T, Liu N, et al. (2009). Distribution and regulation of protein expression of the free fatty acid receptor GPR120. Naunyn Schmiedebergs Arch. Pharmacol. 379: 427-434. http://dx.doi.org/10.1007/s00210-008-0390-8

Oh DY, Talukdar S, Bae EJ, Imamura T, et al. (2010). GPR120 is an omega-3 fatty acid receptor mediating potent antiinflammatory and insulin-sensitizing effects. Cell 142: 687-698. http://dx.doi.org/10.1016/j.cell.2010.07.041

Sakamoto H, Kogo Y, Ohgane J, Hattori N, et al. (2008). Sequential changes in genome-wide DNA methylation status during 
adipocyte differentiation. Biochem. Biophys. Res. Commun. 366: 360-366. http://dx.doi.org/10.1016/j.bbrc.2007.11.137

Schook L, Beattie C, Beever J, Donovan S, et al. (2005). Swine in biomedical research: creating the building blocks of animal models. Anim. Biotechnol. 16: 183-190. http://dx.doi.org/10.1080/10495390500265034

Steneberg P, Rubins N, Bartoov-Shifman R, Walker MD, et al. (2005). The FFA receptor GPR40 links hyperinsulinemia, hepatic steatosis, and impaired glucose homeostasis in mouse. Cell Metab. 1: 245-258. http://dx.doi.org/10.1016/i.cmet.2005.03.007

Zhu JG, Xia L, Ji CB, Zhang CM, et al. (2012). Differential DNA methylation status between human preadipocytes and mature adipocytes. Cell Biochem. Biophys. 63: 1-15. http://dx.doi.org/10.1007/s12013-012-9336-3

\section{Supplementary material}

Table S1. Distribution of the significantly different methylated sites.

http://www.geneticsmr.com/year2016/vol15-1/pdf/gmr4240 supplementary.pdf 